\title{
Diskussionsbeiträge
}

\section{Bewertungsmaßstäbe für die biologische Vielfalt}

\author{
Birgit Georgi ${ }^{1}$
}

Umweltbundesamt, Fachgebiet II 1.1 Grundsatzfragen der Ökologie, Postfach 3300 22, D-14191 Berlin (birgit.georgi@uba.de)

\begin{abstract}
DOI: http://dx.doi.org/10.1065/uwsf2003.01.051
Zusammenfassung. Das wesentliche Ziel des UN-Übereinkommens über die biologische Vielfalt besteht in der Erhaltung der Biodiversität auf der Erde. Da biologische Vielfalt als Ressource - als Nahrungsmittel, Grundstoff für die Industrie, als Sauerstofflieferant, usw. - vom Menschen direkt oder indirekt genutzt wird, ist ihre Nutzung nachhaltig zu gestalten. Um entsprechende Schutz- und Nutzungskonzepte zu entwickeln, bedarf es geeigneter Maßstäbe zur Bemessung der Nachhaltigkeit.

Für den Bereich des Umweltschutzes wurde im Umweltbundesamt analysiert, welche Maßstäbe in den verschiedenen Bereichen derzeit zur Anwendung kommen. Dazu wurden nicht nur die Maßstäbe selbst sondern auch Zusatzinformationen, wie das zugrundeliegende Leitbild, die Berücksichtigung der einzelnen Bestandteile biologischer Vielfalt, ihre Konkretheit und Verbindlichkeit erfasst und ausgewertet. Obwohl Biodiversität als eigenes Schutzgut bisher nur in Ausnahmefällen in die verschiedenen Arbeiten eingegangen ist, existieren bereits eine ganze Reihe von Maßstäben zu Biota, die für das Schutzgut relevant sind. Gleichzeitig sind noch viele Lücken und Inkonsistenzen festzustellen, die in Zusammenarbeit mit anderen Politikbereichen aufzuarbeiten sind, um eine Grundlage für die erwähnten Schutz- und Nutzungskonzepte zu erhalten.
\end{abstract}

Schlagwörter: Biodiversität; biologische Vielfalt; Bewertungsmaßstäbe; ökologische Bewertung; Indikatoren; Übereinkommen über die biologische Vielfalt

\section{Einleitung}

Vor 10 Jahren wurde das UN-Übereinkommen über die biologische Vielfalt (Biodiversitätskonvention) in Rio de Janeiro unterzeichnet. Damit wurde biologische Vielfalt in den Rang eines globalen Schutzgutes mit essentieller Bedeutung für die Menschheit erhoben. Die biologische Vielfalt soll erhalten werden. Schutz und nachhaltige Nutzung sind die beiden Wege, die die Konvention hierfür vorgibt.

Um messen zu können, ob wir mit unseren Bemühungen zur Erhaltung Erfolg haben, benötigen wir Bewertungsmaßstäbe als Messlatte. Viele Akteure - der Tourismus, die Landwirtschaft oder die Siedlungsentwicklung - beeinflussen die biologische Vielfalt. Auch sie benötigen Maßstäbe für die Beurteilung, ob ihr Handeln zum Erhalt der biologischen Vielfalt beiträgt oder das Gegenteil bewirkt. Die folgenden Ausführungen sollen einen Einblick über die aktuelle Situation der Bewertungsmaßstäbe zur biologischen Vielfalt aus dem Blickwinkel des Umweltschutzes geben.

\footnotetext{
${ }^{1}$ Birgit Georgi: 1989 Diplom (Landschaftsarchitektur) Universität Dresden, 1989-1990 wissenschaftliche Mitarbeiterin am Institut für Umweltschutz Berlin, seit 1991 wissenschaftliche Angestellte beim Umweltbundesamt. Themen: ökologische Planung und Bewertung, biologische Vielfalt
}

\begin{abstract}
Evaluation criteria for biological diversity. The main objective of the UN Convention on Biological Diversity is the global conservation of biological diversity. As biological diversity is utilised - directly or indirectly - as a resource for human needs, this utilisation has to be made sustainable. The elaboration of adequate protection and utilisation concepts requires suitable evaluation criteria to measure sustainability.

For the sector of environmental protection, the Federal Environmental Agency has carried out a study to determine the criteria which are currently used in different areas. This study (review and evaluation) covers not only the criteria themselves, but also includes additional information such as the underlying principles.

Biodiversity as an autonomous area of protection has been addressed in only quite a few papers up to now. However, there already exists a range of criteria which are relevant for biodiversity. But at the same time many gaps and inconsistencies were identified which have to be eliminated in cooperation with other policy sectors to obtain a basis for the above-mentioned protection and utilisation concepts.
\end{abstract}

Keywords: Biodiversity; biological diversity; evaluation criteria; ecological evaluation; indicators; Convention on Biological Diversity

\section{Ausgangslage}

Der Begriff der biologischen Vielfalt oder auch Biodiversität wurde Mitte der achtziger Jahre von Biologen geprägt, um den globalen Verlust der Vielfalt und die daraus resultierenden möglichen Gefahren einer breiteren Öffentlichkeit bewusst zumachen. Er umfasst einerseits die gesamte lebende Materie von der Erbinformation (Genom) über die Arten bis hin zu komplexen Ökosystemen und den Prozessen, die darin ablaufen, oder wie in der Biodiversitätskonvention formuliert: "... die Vielfalt innerhalb der Arten, zwischen den Arten und die Vielfalt der Ökosysteme" (UNEP 1992). Zum anderen zielt er auf die Unterschiedlichkeit der biologischen Einheiten.

Die Ausprägung der Biodiversität ist weder weltweit homogen noch über lange Zeiträume gleichbleibend. Die Standort- und Strukturvielfalt unserer Erde bedingt eine in den verschiedenen Regionen stark differenzierte biologische Vielfalt. Die Standortbedingungen können sich zudem im Laufe der Zeit ändern und damit auch die biologische Vielfalt. Beispielsweise kann eine Erwärmung die Abwanderung von kälteliebenden Arten hervorrufen und die veränderte Artenzusammensetzung wiederum eine Änderung des gesamten Ökosystems mit seinen Strukturen und Funktionen. Diese natürliche Dynamik prägt die biologische Vielfalt, deren aktueller Zustand damit immer nur eine Momentaufnahme darstellt. 
Auch wenn eine andauernde Veränderung der Biodiversität naturgegeben ist, gehen Wissenschaftler davon aus, dass die derzeitige Entwicklung massiv vom Handeln des Menschen gesteuert wird. Die geschätzte Aussterberate liegt mehr als zehntausendfach über der natürlichen Rate (Wilson 1995). Dadurch kommt es absolut betrachtet zu einem Verlust an biologischer Vielfalt, dessen Folgen für uns noch weitgehend unklar sind.

Um diesem globalen Problem entgegenzutreten wurde 1992 das Übereinkommen über die biologische Vielfalt verabschiedet und mittlerweile von mehr als 180 Staaten ratifiziert. Es hat zum Ziel, die biologische Vielfalt zu schützen und ihre Nutzung nachhaltig zu gestalten. Aufgrund der räumlich differenzierten Ausprägung der Biodiversität kann die Forderung nach dem Erhalt der biologischen Vielfalt auf der globalen Ebene nur bedingt umgesetzt werden. Um die Ziele zu erreichen, bedarf es vor allem räumlich differenzierter Konzepte, die durch die einzelnen Vertragsstaaten zu erarbeiten sind.

\section{Zielkonzept für die biologische Vielfalt}

Für die national und regional zu erarbeitenden Biodiversitätsstrategien werden neben den globalen auch regionsspezifische Leitbilder benötigt, die angeben, welche Ausprägung der Biodiversität unter Berücksichtigung ihrer natürlichen Dynamik angestrebt wird. Durch Umweltziele und -standards müssen diese Leitbilder weiter konkretisiert werden.

Bei der Formulierung der Zielvorstellungen zur Biodiversität muss beachtet werden, dass nicht nur ein einziger anzustrebender Zustand unserer Umwelt und damit der Biodiversität existiert, sondern mehrere denkbar sind. Wohl muss ein weitgehender Konsens darüber hergestellt werden, welche Grenzen nicht überschritten werden dürfen, um unsere natürlichen Lebensgrundlagen nicht zu zerstören. Innerhalb dieses Rahmens existiert jedoch ein Spielraum, der von der Gesellschaft genutzt werden kann (WBGU 2000).

Von den übergeordneten Leitbildern müssen dann unter $\mathrm{Zu}$ hilfenahme der Wirkungsforschung für einzelne Bereiche und Sektoren konkrete Ziele und Standards abgeleitet werden. Der Vergleich zwischen diesen Zielen und dem ermittelten aktuellen Zustand zeigt an, ob eine Nutzung in bezug auf das Schutzgut Biodiversität nachhaltig erfolgt. So sieht der Wissenschaftliche Beirat für Globale Umweltfragen in seinem Jahresgutachten 2000 ein wesentliches Problem der Umsetzung der Biodiversitätskonvention in Deutschland in dem Fehlen klar quantifizierter Zielvorgaben (WBGU 2001).

Eine Vorgehensweise zur Aufstellung eines Zielkonzepts ist in UBA 2000c (Abb.1) beschrieben. Als Bewertungsmaßstäbe für eine nachhaltige Nutzung dienen Idealerweise quantifizierte Umweltstandards. Wo deren Ableitung nicht möglich ist, müssen Umweltqualitäts- oder Umwelthandlungsziele als Bewertungsmaßstäbe herangezogen werden.

\section{Situation der Bewertungsmaßstäbe}

Auch wenn erst vor etwa 15 Jahren begonnen wurde, das Schutzgut der biologischen Vielfalt zu thematisieren, wurden

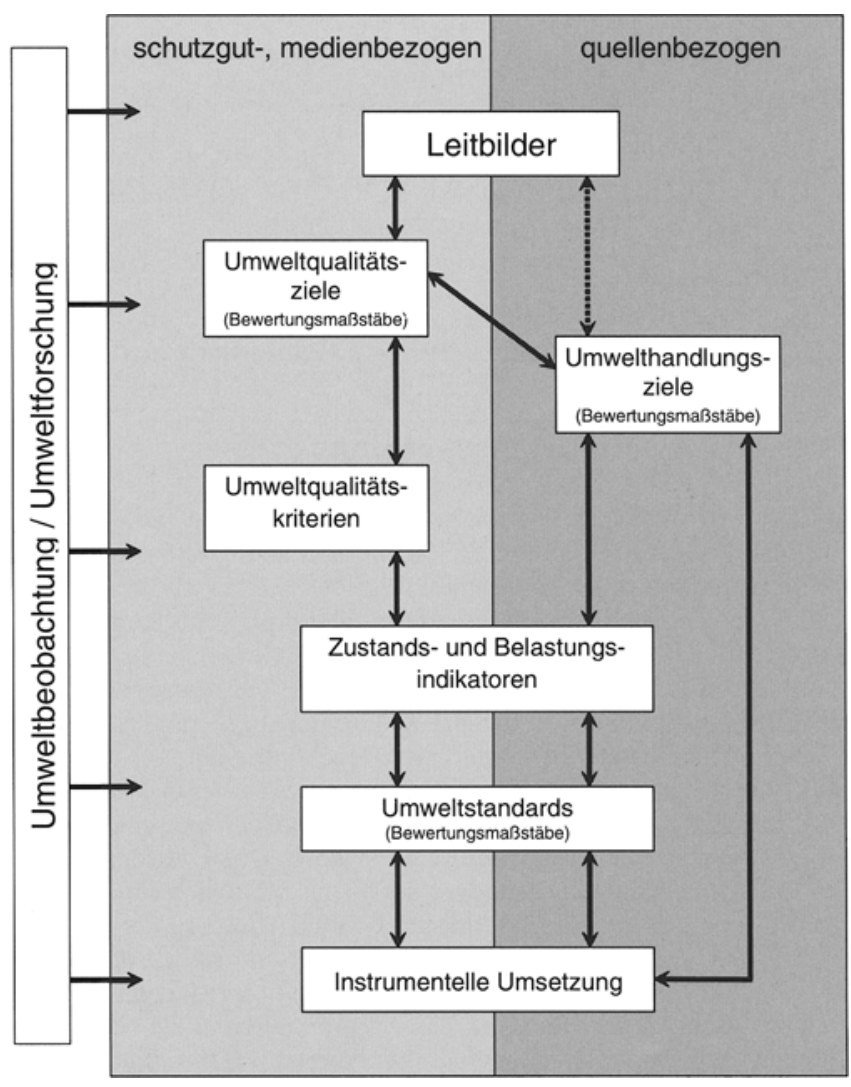

Abb. 1: Leitbildorientierte Formulierung von Umweltqualitäts- und Umwelthandlungszielen (UBA 2000c)

seit langem in Deutschland in vielen Bereichen des Umweltschutzes Bewertungen von Umweltwirkungen auf die Biota durchgeführt. Der Spannungsbogen reicht von Strategien der nachhaltigen Nutzung, wie bei der Nahrungsmittelproduktion oder der Ressourcennutzung, bis hin zur Stoffbewertung oder der Bewertung im Rahmen der Freisetzung von gentechnisch veränderten Organismen.

Die Bewertung erfolgte bisher in der Regel aus der sektoralen Sicht des jeweiligen Bereiches heraus und innerhalb eines definierten Anwendungskontextes. Eine übergreifende Betrachtung wurde nicht eingefordert. Von daher besteht die Gefahr, dass die verschiedenen Vorgehensweisen bzgl. der Bewertung zu Ungenauigkeiten führen und damit möglicherweise zu einem unzureichenden Schutz der Biodiversität.

Für den Bereich des Umweltschutzes stellt sich die aktuelle Situation dar wie in den Abschnitten 3.1 bis 3.6 beschrieben.

\subsection{Berücksichtigung der Elemente der Biodiversität}

Grundsätzlich ist die Biodiversität durch die Berücksichtigung der Biota als Schutzgut in den verschiedenen Gesetzen und Vorschriften des Umweltschutzes enthalten. Dabei wird jedoch der Begriff 'biologische Vielfalt' bisher selten verwendet, wohl aber einzelne Bestandteile oder Synonyme. Beispielhaft gibt Tabelle 1 einen Überblick über ihre Verwendung in den einschlägigen Umweltgesetzen und zeigt die Inhomogenität in der Verwendung der Begriffe. 
Tabelle 1: Wesentliche rechtliche Instrumente des Umweltschutzes, die einen Bezug zu biologischer Vielfalt aufweisen

\begin{tabular}{|c|c|c|c|c|c|c|c|c|c|c|c|}
\hline 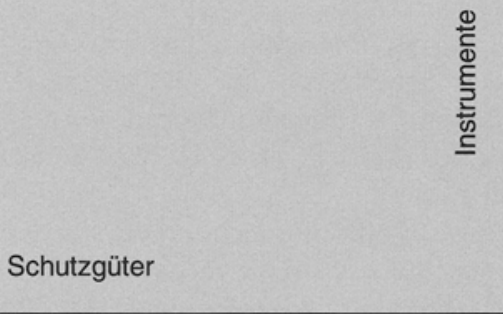 & 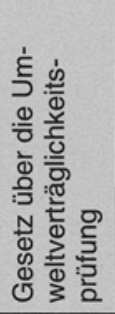 & 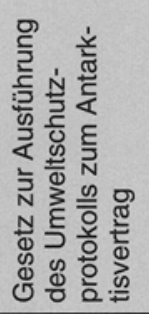 & 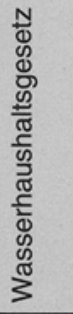 & 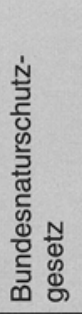 & 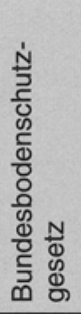 & 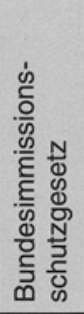 & 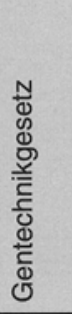 & 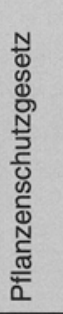 & 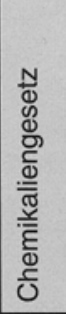 & 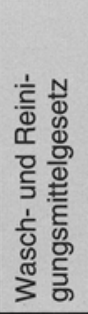 & 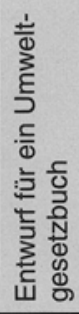 \\
\hline biologische Vielfalt & & & & & & & & & & & $\mathrm{X}$ \\
\hline Tiere & $\mathrm{X}$ & $\mathrm{X}$ & $\mathrm{X}$ & $\mathrm{X}$ & $\mathrm{X}$ & $\mathrm{X}$ & $\mathrm{X}$ & $\mathrm{X}$ & $\mathrm{X}$ & & \\
\hline Pflanzen & $\mathrm{X}$ & $\mathrm{X}$ & $\mathrm{X}$ & $\mathrm{X}$ & $\mathrm{X}$ & $\mathrm{X}$ & $\mathrm{X}$ & $\mathrm{X}$ & $\mathrm{X}$ & & \\
\hline Art & & $\mathrm{X}$ & & $\mathrm{X}$ & & & & & & & \\
\hline Organismus & & & & & $\mathrm{X}$ & & & $\mathrm{X}$ & & & \\
\hline Mikroorganismus & & & & & & & & $\mathrm{X}$ & $\mathrm{X}$ & & \\
\hline genetisch veränderter Organismus & & & & & & & $\mathrm{X}$ & & & & \\
\hline gebietsfremder Organismus & & $\mathrm{X}$ & & $\mathrm{X}$ & & & & $\mathrm{X}$ & & + & \\
\hline Erbinformation (Genom) & & & & & & & $\mathrm{X}$ & $\mathrm{X}$ & $\mathrm{X}$ & & \\
\hline Population & & $\mathrm{X}$ & & & & & & & & & \\
\hline Biotop & & & & $\mathrm{X}$ & & & & & & & \\
\hline Ökosystem & & $\mathrm{X}$ & $\mathrm{X}$ & & $\mathrm{X}$ & & & & & & \\
\hline Lebensgemeinschaft & & & & $\mathrm{X}$ & & & & & & & \\
\hline Naturhaushalt & & & $\mathrm{X}$ & $\mathrm{X}$ & $\mathrm{X}$ & & & $\mathrm{X}$ & $\mathrm{X}$ & $\mathrm{X}$ & \\
\hline Landschaft & $\mathrm{X}$ & & $\mathrm{X}$ & $\mathrm{X}$ & & & & & & & \\
\hline \multicolumn{12}{|l|}{ Vegetationstyp } \\
\hline ökologische Funktionen und Potenziale & & & $\mathrm{X}$ & $\mathrm{X}$ & $X$ & & $X$ & & & & \\
\hline Umwelt & & & & & & & $\mathrm{X}$ & & $\mathrm{X}$ & & \\
\hline
\end{tabular}

Versucht man, die Begriffe den verschiedenen Ebenen der biologischen Vielfalt - Genom, Art, Ökosystem - zuzuordnen, zeigt sich, dass vor allem die Arteneben sowie die ökosystemare Ebene betrachtet werden. Die genetische Diversität wird relativ seiten einbezogen.

Die Funktionen der Biota bei der Steuerung der natürlichen Prozesse und Stoffkreisläufe, welche die Grundlage für die Bereitstellung der unterschiedlichsten Güter und Dienstleistungen von Nahrungsmitteln und Baumaterial bis zum Erhalt der Bodenfruchtbarkeit oder der Selbstreinigung von Gewässern bilden, werden in den Umweltgesetzen und Bewertungsvorschriften derzeit nur teilweise erfasst. Mittelbar ist der Funktionsaspekt in den Bewertungsverfahren enthalten, welche die ökosystemare Ebene berücksichtigen - beispielsweise Gewässerökosysteme im Rahmen der EG-Wasserrahmenrichtlinie. Allerdings ist auch in diesen Fällen die Einbeziehung aller Funktionen nicht automatisch sichergestellt. Dazu muss überprüft werden, ob die der Bewertung zugrundeliegenden Kriterien wie Sauerstoffgehalt, Struktur des Flussbetts oder Abundanz von Arten ausreichen, um alke relevanten Funktionen der Gewässerökosysteme zu beschreiben.

\subsection{Berücksichtigung der Diversität der Biota}

Die Berücksichtigung der einzelnen Bestandteile der Biodiversität an sich, wie Tiere, Pflanzen, Ökosysteme, ist noch nicht gleichzusetzen mit einer systematischen Einbeziehung der Eigenschaft Biodiversität, der Eigenschaft der Biota voneinander verschieden zu sein. Normalerweise müsste eine Bewertung von Umweltwirkungen auf die Biota diese Eigenschaft mit umfassen. In der Realität wird dieser jedoch Aspekt kaum thematisiert oder aber sehr abstrakt behandelt wird.
Bei der Risikoabschätzung von Freisetzungen gentechnisch veränderter Organismen berücksichtigt das Umweltbundesamt zwar die Änderung der biologischen Vielfalt als ein eigenständiges Bewertungskriterium (Quelle: www.umweltbundesamt.de); als Problem erweist sich jedoch, dass der dazugehörige Maßstab nach $\$ 16(1)$ des Gentechnikgesetzes 'keine schädlichen Auswirkungen' nicht hinreichend präzise ist. Für eine qualifizierte Bewertung ist zu klären, welche Veränderungen als Schaden anzusehen sind. Andererseits gibt es eine Reihe von Maßstäben, bei deren Ableitung Biodiversitätsaspekte zwar nicht explizit berücksichtigt wurden, diese aber mit abdecken könnten, was zu überprüfen wäre (Kasten 1).

\begin{abstract}
Gewässerschutz:
Die Zielvorgaben der LAWA für ökotoxikologisch gefährliche Stoffe in Binnengewässern basieren aut der Ermittlung der NOEC von Vertreterarten von vier Trophiestufen unter Anwendung eines Ausgleichfaktors von 0,1-0,01. Zum einen gibt es Anzeichen dafür, dass es in Gewässern Arten gibt, die empfindlicher als die ausgewählten Vertreterarten reagieren und damit bei Zugrundelegung der Maßstäbe nicht geschützt wären, zum anderen sollte entsprechend dem Erkenntnisfortschritt überprüft werden, ob mit den ausgewählten Vertreterarten tatsächlich die Schlüsselarten erfasst wurden, die die Funktionen des Gewässerökosystems dauerhaft aufrecht erhalten.

Stoffgesetze / Pflanzenschutzmittel (EU-RL 91/414/EWG):

Die Auswahl der Testspezies richtet sich nicht nach ihrer ökologischen Relevanz in der Biozönose, sondern nach Kriterien wie Verfügbarkeit, Eignung für Laborversuche und dergleichen. Daher sind die Maßstäbe derzeit in bezug auf die ökosystemare und funktionale Ebene von Biodiversität ebenso wenig ausreichend wie für Aussagen zu gegebenenfalls empfindlicheren Arten als die gewählten Testspezies.
\end{abstract}

Kasten 1: Beispiele für Maßstäbe, die hinsichtlich inrer Weiterentwicklung tür den Diversitätsaspekt überprüft werden müssen 


\subsection{Qualität der vorhandenen Bewertungsmaßstäbe}

Für eine Bewertung im Hinblick auf das Schutzgut der Biota werden Bewertungsmaßstäbe verwendet, die auf die verschiedenen Einflüsse bezug nehmen:

- physikalische (Temperatur, Strukturen, Dichte...),

- chemische (Konzentrationen von Stoffen oder Stoffgemischen) und

- biologische (Artenzusammensetzung, Biotopausstattung, Zufuhr oder Entnahme von Organismen...) oder

- Kombinationen physikalischer, chemischer oder biologischer Einflüsse.

Messbare quantitativen Standards (im Sinne von Zielvorgaben oder Grenzwerten) findet man relativ zahlreich im stofflichen Bereich, bei der Bewertung im Rahmen der Stoffgesetze oder der Umweltqualität in den Medien Wasser, Boden und Luft - den traditionellen Umweltschutzbereichen. Selten werden jedoch Kombinationswirkungen berücksichtigt. Ein positives Beispiel hierfür sind die Critical Loads, die sich auf die komplexen Prozesse Versauerung und Eutrophierung beziehen: Schwefeldioxid und Stickoxide haben ebenso wie Ammoniak mit ihrem Eintrag über den Bodenpfad eine versauernde Wirkung auf Ökosysteme. Stickoxide und Ammoniak wirken zusätzlich eutrophierend.

Ursache für eher qualitative Formulierungen sind zum einen Teil Wissensdefizite, zum anderen Teil ein fehlender Konsens zur Höhe des akzeptablen Risikos in bezug auf die Biodiversität. Sehr abstrakte Maßstäbe, wie z.B. die Mehrung des biologischen Abbau- und Aufbaupotentials und die Unterstützung des Selbstregulationsvermögens biologisch aktiver, ausreichend mit organischer Masse versorgter Böden durch Mikroorganismen und Bodentiere entsprechend der 'Guten fachlichen Praxis der landwirtschaftlichen Bodennutzung' (Bundesanzeiger Nr. 73 vom 20.04.1999), erschweren in der Praxis die Bewertung. Für eine bessere Anwendbarkeit ist daher ihre weitere Ausdifferenzierung in einzelne, möglichst quantitative Parameter notwendig, die zusammengenommen den angestrebten Zustand beschreiben.

Die rechtliche Verbindlichkeit der Maßstäbe variiert stark. Nur einige sind rechtlich festgelegt. Diese wiederum stellen nahezu ausschließlich Vorsorge- und Prüfwerte im stofflichen Bereich dar, müssen also nicht zwingend eingehalten werden oder sind noch, wie bereits erläutert, sehr weich formuliert. Doch nicht immer erscheint eine rechtliche Festlegung der Bewertungsmaßstäbe sinnvoll und wünschenswert. Mitunter werden Bewertungsmaßstäbe mittelbar verbindlich, so z.B. im Falle der Critical Loads für Versauerung und Eutrophierung, die als Basis für die Berechnung für verbindliche nationale Emissionsobergrenzen dienen. In anderen Fällen wie im Meeresschutz - erscheint es nicht möglich, Wirkungsschwellen zu definieren. Hier sollte eine Reduzierung der Einwirkungen soweit wie möglich angestrebt werden.

\subsection{Ableitung der Maßstäbe}

Die Bewertungsmaßstäbe unterscheiden sich weiterhin in der Art ihrer Ableitung je nach Anwendungsbereich. Sie lassen sich in drei Gruppen zusammenfassen:

1. Maßstäbe, die Festlegungen für direkte Einwirkungen darstellen, teilweise ohne dabei zunächst die tatsächlichen Belastungen in der Umwelt zu berücksichtigen, wie die Stoffbewertung, die Freisetzung gentechnisch veränder- ter Organismen, die Aussetzung gebietsfremder Arten, die direkte Entnahme von Biota,

2. Maßstäbe, welche die abiotische und biotische Umweltqualität der Umweltmedien beschreiben, die zum Schutz der Biodiversität notwendig ist, wie Umweltqualitätsziele / -standards für Luft, Klima, Binnengewässer, Meeresgewässer, Boden, Grundwasser und

3. Maßstäbe, die in bezug auf Nutzungen definiert werden, welche entweder direkt Einfluss auf die Biota haben oder indirekt über die Qualität der Umweltmedien wirken, wie Umwelthandlungsziele und -standards für die Land-, Forstwirtschaft, Fischerei, Siedlung, Verkehr, Industrie und den Tourismus.

Wenn diese Maßstäbe in der Vergangenheit in der Regel auch sektoral und separat abgeleitet wurden, so bestehen dennoch zwischen diesen Gruppen faktisch zahlreiche Querverbindungen. So können die auf die ökotoxikologische Wirkungsforschung gestützten Bewertungsmaßstäbe (a) in bezug auf das Schutzgut der Biodiversität nur ihre Wirkungen entfalten, wenn sie durch Maßstäbe zur Umweltqualität (b) und zur nachhaltigen Nutzung (c) ergänzt werden (siehe auch Kasten 2).

Allein die Anwendung eines nach EU-RL 91/414/EWG zugelassenen
Pflanzenschutzmittels stellt noch nicht sicher, dass keine unannehm-
baren Auswirkungen auf die in der RL beschriebenen (biotischen)
Schutzgüter erfolgen. Dazu bedarf es ergänzend einer be-
stimmungsgerechten und sachgerechten Anwendung. Dies erfordert
eine enge Zusammenarbeit zwischen den Institutionen, die die Be-
wertung der Pflanzenschutzmittel vornehmen und den entsprechen-
den landwirtschaftlichen Institutionen. Die Grundsätze hierzu wer-
den in der, guten fachlichen Praxis im Pflanzenschutz' (Ausführungs-
bekanntmachung im Bundesanzeiger Nr. 220a vom 21.11.1998)
beschrieben. Allerdings reicht der dort genannte Maßstab ,keine
schädlichen Auswirkungen auf die Gesundheit von Mensch und Tier
und auf das Grundwasser und keine sonstigen nicht vertretbaren
Auswirkungen, insbesondere auf den Naturhaushalt' noch nicht aus,
da noch nicht genauer definiert ist, ab wann von schädlichen oder
nichtvertretbaren Auswirkungen auszugehen ist.

Kasten 2: Verknüpfung der verschiedenen Ansätze für die Ableitung von Bewertungsmaßstäben: Beispiel Pflanzenschutzmittel

\subsection{Zugrundeliegendes Leitbild}

Obwohl die Formulierung spezifischer Bewertungsmaßstäbe für die einzelnen Handlungsbereiche sinnvoll und wünschenswert ist - denn dies erhöht ihre praktische Anwendbarkeit so ist doch zu beachten, dass es sich um ein und dasselbe Schutzgut - die Biodiversität - handelt. Will man hinsichtlich ihres Erhalts erfolgreich sein, ist es daher notwendig, die einzelnen Bewertungsmaßstäbe einem Biodiversitätsleitbild folgend aufeinander abzustimmen und ein konsistentes Konzept zu bilden. Die grundsätzliche Vorgehensweise für die Ableitung eines solchen Zielkonzepts ist in Abb. 1 dargestellt.

Es existiert derzeit kein abgestimmtes, ausreichend konkretes und umfassendes Leitbild auf der nationalen Ebene. Oftmals wurde bei der Entwicklung von Vorschriften und Richtlinien unbewusst die Wertvorstellung zur biologischen Vielfalt des diese Texte Erarbeitenden als Biodiversitätsleitbild zugrundegelegt. In den Vorschriften und dazugehörigen Erläuterungen ist dieses Leitbild nur selten explizit beschrieben. Dies führte zu einer Leitbildvielfalt (Kasten 3 ).

Zum Teil weisen die Leitbilder Ähnlichkeiten auf oder sind Teilleitbilder. In einer Reihe von Fällen sind aber Zielkonflikte ab- 
- Erhalt des derzeitigen Zustandes einschließlich der Entwicklungsmöglichkeiten

- verschiedene (historische oder anderweitige) Referenzzustände

- Erhalt (und Entwicklung) natürlicher Funktionen

- Erhalt / Wiederherstellung eines natürlichen und sich selbst erhaltenden Ökosystems, in dem Prozesse ungestört ablaufen können

- Erhalt / Wiederherstellung einer weitgehend naturnahen biotischen Ausstattung

- Erhalt/Wiederherstellung eines weitgehend unbeeinflussten Zustandes oder Vermeidung von Beeinträchtigungen

- Erhalt der heimischen Biodiversität

- Erhalt / Wiederherstellung des heutigen potentiellen und naturraumtypischen Zustandes

- Erhalt und Wiederherstellung des guten Zustandes (geringfügige anthropogene Beeinträchtigung)

- Erhalt / Wiederherstellung der potentiellen natürlichen Vegetation

- Erhalt eines langfristig nachhaltigen steady state

- Erhalt von Struktur und Funktion von Ökosystemen

- Erhalt aller Arten oder Biotoptypen

Kasten 3: Auswahl von Biodiversitätsleitbildern in diversen Umweltvorschriften, -gesetzen, Programmen und Plänen

lesbar. Diese könnten durch eine räumliche Entflechtung gelöst werden. Es bedarf daher eines gesellschaftlichen Diskurses zur Leitbildfindung. Er sollte zur Findung eines nationalen Leitbildes führen, welches durch eine regionale und inhaltliche $\mathrm{Zu}$ weisung verschiedener Teilleitbilder zusammengesetzt wird.

\subsection{Zu akzeptierendes Risiko}

Das zu akzeptierende Risiko in bezug auf Biodiversität umfasst Festlegungen, bis zu welchem Niveau ein Risiko noch als vertretbar angesehen werden kann.

In vielen Bereichen ist es noch nicht gelungen, das zu akzeptierende Risikoniveau zu formulieren. So sollen laut Gentechnikgesetz $\$ 16$ unvertretbare schädliche Einwirkungen vermieden werden, jedoch sind keine Kriterien für die Vertretbarkeit vorgegeben. Bei den Zielvorgaben für gefährliche Stoffe in Gewässern behilft man sich mit Sicherheitsfaktoren (UBA 2000b). Im Rahmen der Ausführung des Umweltschutzprotokolls zum Antarktisvertrag versucht man das Risiko durch Setzungen auf der Basis von Expertenwissen zu definieren (UBA 1999). Beispielsweise wird hier die Zerstörung von 50\% einer lokalen Population verbreiteter Pflanzenarten und bei seltenen Pflanzenarten von 5\% noch als akzeptabel angesehen. Doch auch diese Ansätze sind bisher weder untereinander konsistent noch wissenschaftlich dahingehend abgesichert, dass das jeweils angestrebte Niveau erreicht wird.

\section{Schlussfolgerungen}

Zusammenfassend kann festgestellt werden, dass bereits eine Vielzahl von Bewertungskriterien und -maßstäben mit bezug zur Biodiversität existiert. Allerdings weisen sie noch verschiedene Fehlstellen und Inkonsistenten auf, die aufgearbeitet werden müssen:

1. Die Begriffsvielfalt zum Schutzgut Biodiversität (einzelne Bestandteile und Synonyme) in rechtlichen Vorschriften kann verwirren, so dass eine klare und einheitliche Benennung und Ausdifferenzierung des Schutzgutes Biodiversität im Rahmen der Vorschriften dringend notwendig erscheint, um sicherzustellen, dass es umfassend und adäquat berücksichtigt wird.
2. Die Betrachtung der verschiedenen Bestandteile und Aspekte der Biodiversität erfolgt bisher nicht gleichermaßen. Die vorhandenen Lücken, insbesondere auf der Genomebene und bei den Funktionen, müssen geschlossen werden.

3. Nur in Ausnahmefällen ist die Eigenschaft Diversität der Biota explizit berücksichtigt. Die übrigen Maßstäbe sollten dahingehend überprüft und gegebenenfalls weiterentwickelt werden.

4. Die Qualität einer Reihe von Bewertungsmaßstäben erscheint noch nicht ausreichend. Rein qualitative und undifferenzierte Maßstäbe sind in der Praxis nur schwer handhabbar und bedürfen daher einer weiteren Konkretisierung. Gegebenenfalls ist dazu weitere Wirkungsforschung notwendig. Zudem sollten neben stofflichen Einwirkungen auch physikalische und biologische verstärkt untersucht werden.

5. Die sektoral abgeleiteten Maßstäbe sollten in den Zusammenhang mit anderen Bereichen gestellt und bezüglich der Konflikte und Lücken analysiert und aufgearbeitet werden.

6. Die Inkonsistenzen und Konflikte zwischen den unterschiedlichen sektoral abgeleiteten Leitbildern müssen gelöst werden, beispielsweise durch die Festlegung regional unterschiedlicher (Teil-) Leitbilder.

7. Ein Konsens über die Einstufung einer Veränderung als Schaden für die Biodiversität und über das zu akzeptierende Risiko muss ähnlich dem Vorgehen beim Schutzgut der menschlichen Gesundheit durch eine gesellschaftliche Risikodiskussion erreicht werden.

Die Aufarbeitung dieser Punkte muss gemeinsam mit allen Akteuren im Themenfeld Biodiversität - nicht nur Umweltund Naturschutz - erfolgen. Einen guten Ansatzpunkt für die Aufarbeitung böte der Prozess der Entwicklung einer nationalen Biodiversitätsstrategie, wie sie das Übereinkommen über die biologische Vielfalt von seinen Vertragsstaaten fordert.

\section{Literatur}

UBA - Umweltbundesamt (Hrsg.) (1999): Entscheidungshilfen für die Genehmigungspraxis zur Umsetzung des Gesetzes zur Ausführung des Umweltschutzprotokolls vom 4. Oktober 1991 zum Antarktisvertrag (AUG). UBA-Texte 16/99, Berlin

UBA - Umweltbundesamt (Hrsg.) (2000a): Bodenbiologische Güteklassen. UBA-Texte $6 / 00$, Berlin

UBA - Umweltbundesamt (Hrsg.) (2000b): Umweltqualitätsziele für gefährliche Stoffe in Gewässern - Internationaler Vergleich der Ableitungsmethoden. UBA-Texte 24/00, Berlin

UBA - Umweltbundesamt (Hrsg.) (2000c): Ziele für die Umweltqualität - Eine Bestandsaufnahme. Verlag: Erich Schmidt, Berlin

UBA - Umweltbundesamt (Hrsg.) (2001): Nachhaltigkeit und Vorsorge bei der Risikobewertung und beim Risikomanagement von Chemikalien. UBA-Texte 30/01, Berlin

UNEP (1992): Übereinkommen über die biologische Vielfalt. Rio de Janeiro Wilson EO (1995): Der Wert der Vielfalt: Die Bedrohung des Artenreichtums und das Überleben des Menschen. München: Piper, $512 \mathrm{~S}$.

WBGU - Wissenschaftlicher Beirat der Bundesregierung Globale Umweltveränderungen (2000): Welt im Wandel: Erhaltung und nachhaltige Nutzung der Biosphäre - Jahresgutachten 1999. SpringerVerlag Berlin, Heidelberg

WBGU - Wissenschaftlicher Beirat der Bundesregierung Globale Umweltveränderungen (2001): Welt im Wandel: Globale Institutionen für die Umwelt - Jahresgutachten 2000. Springer-Verlag Berlin, Heidelberg

Eingegangen: 24. September 2002 Akzeptiert: 24. Januar 2003 OnlineFirst: 25. Januar 2003 